\title{
Strategies for Regional Mental Health Education under the Influence of the COVID-19 Epidemic: A Case Study of Nanjing, China
}

\author{
Jing Lin \\ Nanjing Research Center of Education Science, Nanjing 210000, Jiangsu, \\ China
}

\begin{abstract}
The outbreak of COVID-19 pneumonia severely affected the psychological health of children and produced negative effects on it. How to help children overcome the negative psychological impact caused by the epidemic is a common concern. During the epidemic, the administrative department of education in Nanjing, China actively carried out psychological health education and assistance for students. I outline the measures for developing mental health and psychological assistance in Nanjing, China.
\end{abstract}

Sci Insigt Edu Front 2020; 5(2):541-545.

Doi: 10.15354/sief.20.rp014

Keywords: COVID-19 Pneumonia Epidemic; Mental Health Education; Psychological Assistance; Mental Health Cloud Platform; Elementary and

Middle School Students

Correspondence to: Jing Lin, Nanjing Research Center of Education Science, Nanjing 210000, Jiangsu, China; Email: 952198256@qq.com.

Conflict of Interests: None. 
$\mathrm{I}$ $\mathrm{N}$ the Spring Festival of 2020, the COVID-19 epidemic broke out and it was very aggressive. China has implemented strong prevention and control strategies. After the holidays, according to the development of the epidemic, Jiangsu Province issued guidance on February 6, 2020 to postpone the start of the school. The epidemic affects everyone's life and learning, and inevitably invades people's spiritual world. As the sense of control is destroyed, everyone generally experiences negative emotions such as panic, helplessness, anxiety, fear, worry, and anger. If a loved one dies during the epidemic, the sadness and feeling of loss will be worsened. Faced with this kind of psychological crisis, as the hub of mental health education guidance and management in Nanjing, Nanjing Mental Health Education Guidance Center of elementary and middle schools responded quickly and launched a series of coping strategies.

\section{Launch of Volunteer Service Mode to Provide Psycho- logical Assistance by Hotline}

Due to the highly contagious nature of the COVID-19 virus, daily face-to-face mental health education and consultation activities cannot be carried out normally, and the hotline has become the best carrier for psychological assistance. Nanjing has always had a team of psychological volunteers, who carry out psychological hotline services at the Nanjing Elementary and Middle School Students' Psychological Assistance Center (Teacher Tao Workstation). At the same time, the hotlines of the 12 district-level Tao teacher workstations in Nanjing were opened immediately during the epidemic, and the staff on duty came to work under strict protection to provide psychological help for the hotline. In addition, Nanjing "Professor Ning" Studio, a teacher's mental health service platform, has also been launched to provide teachers with psychological anti-epidemic guidance. The opening of these hotlines and consulting platforms has sent psychological support and spiritual comfort to elementary and middle school students, parents, and teachers in Nanjing.

In the early stage of the epidemic, the content of the hotline was mainly about: fear, worry and anxiety of the epidemic; concerns about the safety of themselves and their families; and questions about the disease itself, such as whether the symptoms of a cold and fever are infected with COVID-19 pneumonia, etc. At this time, the function of the hotline is to popularize science, stabilize emotions, and find positive resources to respond.

In the middle stage of the epidemic, when everyone gradually understood the development of the epidemic and the country's response plan, the panic mentality turned to adaptation and began to focus on the topics of family life and interpersonal conflict. Because children have this extra long vacation, the time between parents and children has increased. The benefit of this is that the children have a companionship that is usually difficult to obtain; on the other hand, it also potentially compresses the parents and children time and space to get along. As a result, conflicts between families with poor relationship and conflicts have worsened. At this stage, the hotline is mainly 
to guide the caller's emotions and guide them to learn good interpersonal communication methods.

In the late stage of the epidemic, children's long-term exposure to electronic products led to some overuse of the Internet, which stimulated conflicts between parents and children in the use of electronic products and learning anxiety, especially students who are nearing graduation, such as 8th and 12th graders, talked about their anxiety and helplessness about learning in the hotline. The hotline at this time provided more psychological support and problem-solving strategies to help callers perform psychological fitness and restart the chapter of life.

\section{Compile Guidance Manuals on "Psychological Anti- Epidemic" For Elementary and Middle School Stu- dents, and Give Guidance to Students and Families on Mental Health Education}

Facing the epidemic, the Nanjing Municipal Government and the Nanjing Education Bureau attached great importance to quickly organizing front-line mental health education experts and teachers, combing the psychological hotline at the "Teacher Tao" workstation (including each branch station), and the network psychology of "Professor Ning" Studio psychological distress faced by students, parents, and teachers learned during the consultation. They compiled a set of "Nanjing Primary and Secondary School" Psychological Anti-epidemic "Education Guidebook". Through vivid and easyto-understand methods such as problem descriptions and case analysis, students, parents and teachers were provided with effective and effective anti-epidemic psychological masks to protect the mental health of children and adolescents during the epidemic.

According to the characteristics and needs of different groups of people, this instruction manual is divided into "student part", "parent part" and "teacher part". Among them, the "student part" focuses on guiding children with different family environments to adjust their emotions, manage time effectively, get along with their families, and understand the impact of the epidemic. Look at the problem from a positive perspective and turn the crisis into an opportunity for self-growth. The "parent part" is to guide parents to review the family's upbringing style, life issues and relationship model through the change of family lifestyle caused by the epidemic. By adjusting the family system and parent-child relationship, psychological harmony in family life is achieved. The "teacher part" introduces the concepts and skills of mental health education to teachers, improves teachers' mental health education literacy, promotes them to better understand the psychological needs of students, and to be an effective teacher. This instruction manual is distributed electronically to elementary and middle schools and families for free. 


\section{Design Mental Health Education Courses According to Different Stages of the Epidemic and Carry out Online Course Teaching}

In accordance with the general guidelines of "School's Out, But Class's On", relying on the electrification education resources and "Jinling Micro School" network platform, a series of online courses have been established. According to the different stages of the epidemic, it is divided into: psychological popularization and home instruction courses in the early stage of the epidemic; life education courses in the middle stage of the epidemic; psychological adaptation courses in the late stage of the epidemic, preparing for the start of school.

The specific course content includes: how primary and secondary school students cope with the crisis and challenges brought by the epidemic; how to carry out time management and career planning; how to improve resistance to adversity; how to debug the parent-child relationship of families; how to respond to the requirements of online learning, and how to avoid the risk of Internet addiction; how to maintain brain health during an epidemic; how to respond to various emotional and emotional reactions during the epidemic; and how to work regularly and psychological self-protection during the epidemic.

These courses are recorded by teachers of psychology and arranged into the schedule of online learning for students. The network platform and TV broadcast were used to push resources to families in Nanjing, and class schedules were posted by the homeroom teacher to remind parents to assist their children in learning. These courses have helped students and parents and teachers to provide scientific guidance in coping with the psychological effects of the COVID-19 epidemic, and are generally welcomed.

\section{Relying on Social Networking Platforms to Carry out Home Accompany and Guidance}

During the outbreak, family education needs guidance. Under the organization and recruitment of the Nanjing Women's Federation, Nanjing's psychological volunteer teachers established trust and cooperation with their mothers in the WeChat group. We used the online log-in card method. Every day, parents wrote a detailed story and feelings in family education after work, and the co-operative family education instructor responded, paid attention to and gave targeted guidance to this story.

The guiding principles are affirmative, supportive, and constructive suggestions to help parents see the impact of education methods in interacting with children on children and adolescents. It pays attention to the subtle development and changes in parentchild relationship, whether the needs and expectations of children and adolescents are better seen and treated, and the emotional development and psychological needs of parents in the process of educating children. This "family support plan" has the main goals 
of adjusting family relationships, assisting parents to deal with problem behaviors of children and adolescents, and inspiring parents' self-awareness and growth through the impact on parents' educational concepts and methods. This project cycle has 21 days that helps to form a new family education model.

In summary, what people need in the face of an epidemic are protection, confidence, and caring actions. Nanjing is well-known as the "City of Fraternity and Love". The common concern for the fate of humankind has enabled everyone in it to inspire the potential of life and the spirit of contributing to society. This kind of selfless dedication and service, and the love for children and adolescents, are the greatest motivation to guide us to defeat the epidemic, build up hope, and reshape new life. 